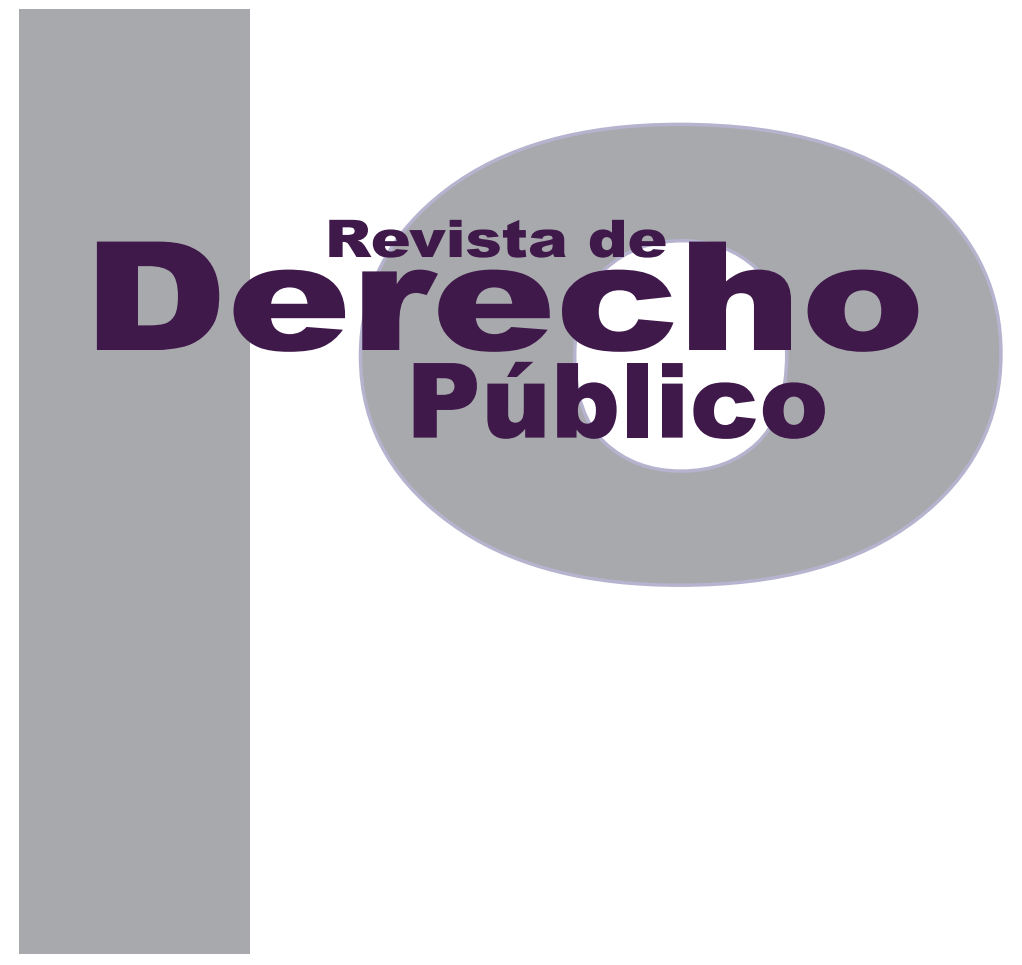

\title{
INTRODUCCIÓN A LA NUEVA FRONTERA HUMANA SOBRE LA DELIMITACIÓN DEL ESPACIO ULTRATERRESTRE Y LOS ASPECTOS RELATIVOS A LA ÓRBITA DE LOS SATÉLITES GEOESTACIONARIOS
}

LINA MARgarita BALlestas ChIRIví

Universidad de los Andes

Facultad de Derecho

Revista de Derecho Público N. ${ }^{\circ} 28$

Enero - Junio de 2012. ISSN 1909-7778 


\title{
INTRODUCCIÓN A LA NUEVA FRONTERA \\ HUMANA \\ Sobre la delimitación del espacio ultraterrestre y los aspectos relativos a la órbita de los satélites geoestacionarios
}

\author{
Lina Margarita Ballestas Chiriví ${ }^{1}$
}

\begin{abstract}
RESUMEN
Hace más de 25 años que la conquista del espacio dejó de hacer parte de la ciencia ficción abriendo las puertas a nuevas relaciones jurídicas, cuestionamientos e instituciones que hoy en día conforman el Derecho del Espacio Ultraterrestre, el cual está basado en dos elementos que en otros campos son tan solo aspiraciones: la paz y el bienestar común. Entre debates caducos, ignorancia y malinterpretaciones, esta rama del Derecho Internacional se enfrenta a la constante evolución científico-técnica sin un desarrollo jurídico equiparable, con una delimitación aún ambigua de su campo de acción, y recursos de particular naturaleza como lo es la órbita geoestacionaria. En este nuevo horizonte jurídico, presto en su verdor a la experimentación e innovación, Colombia es ejemplo tanto de progreso como de entorpecimiento.
\end{abstract}

Palabras clave: Derecho Espacial. Derecho Constitucional. Espacio ultraterrestre. Soberanía. Órbita geoestacionaria.
Over 25 years ago, the idea of space travel went from being science fiction to science fact, thus giving rise to new legal relationships, paradigms, and institutions that would later form the basis for Space Law, which in turn is based on two concepts that are mere aspirations in other fields: peace and the common good. Due to stale debates, ignorance and misinterpretations, this branch of International Law is unable to keep up with scientific progress, with an undefined field of action and resources of a very particular nature, such as the geostationary orbit. In this new legal horizon, prone to experimentation and innovation, Colombia is both a beacon of progress and obstruction.

Keywords: Space Law. Constitutional Law. Sovereignty. Outer Space. Geostationary Earth Orbit.

1 Estudiante de pregrado del doble programa de Derecho e Historia de la Universidad de los Andes. Correo electrónico: lin-ball@uniandes.edu.co 


\section{SUMARIO}

Introducción: la historia del hombre y la conquista del espacio - I. DEFINICIÓN Y DELIMITACIÓN DEL ESPACIO ULTRATERRESTRE - A. El límite Superior - B. El límite inferior: la diferencia entre aire y espacio - 1. La navegación vs. el desplazamiento - 2. La aplicación del concepto de soberanía 3. Propuestas de delimitación - 3.1 Criterios geográfico-científicos - 3.2 Criterios funcionales - II. ASPECTOS RELATIVOS A LA ÓRBITA DE LOS SATÉLITES GEOESTACIONARIOS - 1. Órbita - 2. Los satélites - 3. El espectro electromagnético - A. De soberanía a acceso equitativo - B. El dilema del artículo 101 de la Carta Política - III. CONCLUSIÓN: el espacio en la construcción de un nuevo mundo. 


\section{Introducción. La historia del hombre y la conquista del espacio}

El espacio ha ejercido una especial atracción en el ser humano desde que éste elevó por primera vez su mirada y quedó maravillado por el infinito que se abría ante sus ojos. En un principio, como no poseía el conocimiento para explicarlo, le dio un orden y una estructura a través de mitos y leyendas. Por medio de la observación trazó el mapa de las estrellas y planetas y evidenció su movimiento, incluso sin comprenderlo aún. Esto le permitió medir distancias, guiarse por tierra y navegar por los mares, calcular el paso del tiempo y crear calendarios. Los avances en matemática y el desarrollo del empirismo explicaron el movimiento de los planetas, estableciendo que la Tierra no era el centro del universo. Una a una las verdades fueron revelándose, y grandes mentes desde Anaxágoras hasta Einstein, pasando por Copérnico, Galileo, Kepler y Newton, aportaron en esta conquista teórica del espacio; el dominio real del mismo nunca ha dejado de ser uno de los más grandes sueños de la humanidad.

Tal como en el mito de Ícaro, quien con un par de alas de cera intentó acercarse al Sol, el hombre ha imaginado miles de formas para superar sus limitaciones físicas y explorar el espacio. Ya en la antigua Grecia, Luciano de Samosata relataba cómo pudo llegar a la Luna a través de un tornado que lo impulsó luego de cruzar las Columnas de Hércules, hoy conocidas como el estrecho de Gibraltar ${ }^{2}$. Desde entonces abundaron los

2 El texto donde Luciano de Samosata relata dicha travesía es la sátira Historia Verdadera, en la que se burla de los historiadores, en especial relatos de viajes espaciales, especialmente a la Luna, que tuvieron su apogeo durante los siglos XVI y XVII, época en la que no sólo aumentó la frecuencia de estas travesías literarias, sino que se nutrieron de ciencia al combinar elementos científicos con imaginarios, creando el género conocido como ciencia ficción ${ }^{3}$. En 1516, Ludovico Ariosto escribió el poema Orlando el Furioso, en el que uno de sus personajes viaja a ese mismo cuerpo celeste. En 1605, Cyrano de Bergerac dio a luz la novela Historia cómica de los estados e imperios de la Luna, seguida en 1662 por Historia cómica de los estados e imperios del Sol. El barón de Münchhausen, en los relatos de sus propias aventuras incluyó un paseo lunar, historia que insertó Rudolf Erich Raspe en su libro de 1785: Hazañas extraordinarias y extravagantes. Tomás Campanella, John Wilkins y Francis Godwin, entre otros, escribieron también sobre travesías al espacio. El mismo Kepler, mientras investigaba y planteaba sus leyes del movimiento planetario, escribió en 1623 la que actualmente se considera la primera obra de ciencia ficción, una novela titulada El sueño astronómico, en la que un personaje

\footnotetext{
de Heródoto. El libro cuenta una serie de viajes fantásticos que terminan llevándolo a él y a sus compañeros a la Luna, lugar en el que conocen diversas especies maravillosas, que describe en detalle. La sátira está en el relato mismo, puesto que critica la forma como Heródoto y sus compañeros historiadores dan prevalencia a lo fantástico sobre lo real. Las obras completas de este autor pueden leerse gratuitamente en diversos portales, entre los que se encuentra Google y su servicio Google Books.

"Ciencia ficción es un género de narraciones imaginarias que no pueden darse en el mundo que conocemos, debido a una transformación del escenario narrativo, basado en una alteración de coordenadas científicas, espaciales, temporales, sociales o descriptivas, pero de tal modo que lo relatado es aceptable como especulación racional". SÁNCHEZ, Guillermo, GALLEGO, Eduardo. "Qué es la ciencia-ficción?" (en línea). Disponible en: http://www.ciencia-ficcion.com/opinion/op00842.htm\# (Consulta: 11 de diciembre de 2011).
} 
denominado Duracotus viaja a la Luna y conoce una colonia de seres lunares ${ }^{4}$.

Los relatos más conocidos sobre el viaje lunar y espacial fueron escritos en el siglo XIX por el famoso escritor Julio Verne. En 1865 Verne publicó De la Tierra a la Luna, seguido cuatro años después por el menos famoso Alrededor de la Luna, los cuales servirían de inspiración en años posteriores tanto para artistas como para científicos ${ }^{5}$. En este mismo siglo escribieron sobre el tema Alejandro Dumas y Edgar Allan Poe. Pero fue a comienzos del siglo XX, cuando la tecnología y la ciencia avanzaban a gran velocidad y la aeronáutica era ya un hecho, que aparecieron los tres grandes autores de ciencia ficción, quienes influyeron de formas insospechadas en la realización del sueño de viajar al espacio: el soviético Isaac Asimov, el estadounidense Robert A. Heinlein, y el inglés Arthur C. Clarke. Estos científicos y literatos, además de publicar ensayos y artículos propiamente científico-técnicos, combinaron en sus textos narrativos de forma rigurosa el conocimiento y la innovación científica. Los tres tuvieron la fortuna de vivir la realización de los sueños de todos sus antecesores,

4 Para más sobre la historia de la ciencia ficción y su relación con la odisea espacial, se recomienda ver JAMESON, Fredic. Arqueologías del futuro. El deseo llamado utopía y otras aproximaciones de ciencia ficción. Madrid: Akal, 2009.

5 Julio Verne anticipó en sus relatos los helicópteros, las naves espaciales, las armas de destrucción masiva, los submarinos, el ascensor e incluso el internet. Sus historias inspiraron a los científicos a explorar las posibilidades de hacer de estos elementos realidades tangibles y a dedicar sus vidas a la exploración del espacio. Es bien conocido que Yuri Gagarin afirmó haberse dedicado a la astronáutica gracias a la obra de Verne. Por su parte, las artes han visto en sus obras una fructífera fuente de inspiración, como lo demuestran las múltiples referencias en otras creaciones literarias, y en adaptaciones para cine y televisión. En la segunda mitad del siglo XX, fueron programas de televisión de ciencia ficción como Star Trek los que influyeron en la creación de avances como la telefonía celular. en especial autores y científicos, cuando el 12 de abril de 1961 Yuri Gagarin se convirtió en el primer hombre en viajar al espacio, y ocho años después, el 21 de julio de 1969, Neil Armstrong dio el primer paso sobre la superficie lunar.

De este punto en adelante, los sueños del hombre de conocer y recorrer el espacio dejaron de ser ficción para dar paso a realidades llenas de aventuras maravillosas que llegan a sobrepasar la imaginación: explorar la frontera de nuestro sistema solar, observar rincones a años luz de distancia, crear estaciones espaciales internacionales, comunicar a un planeta entero, llegar a Marte... Y si bien el infinito sigue planteando miles de cuestionamientos que mantienen al ser humano activo, deseoso y expectante, nunca ha estado el hombre más cerca de comprenderlo que hoy. ¿Cuál es el límite del universo? ¿Qué maravillas oculta? ¿Es el hombre la única especie inteligente que lo habita? Y sea o no así, ¿cuál es el rol del ser humano en el universo?

El presente artículo no pretende responder a estos interrogantes; esa labor le corresponde a la ciencia. A nosotros, en el campo del Derecho, sólo nos incumbe tratar el tema de las relaciones jurídicas derivadas de este nuevo horizonte. No significa que esto sea irrelevante; como en cualquier aspecto de la vida humana, el Derecho debe ir de la mano con el hombre, pues posee el poder de evitar conflictos y de solucionar los que se presentan. De otra parte, al ser un territorio nuevo, el espacio le permite al Derecho la posibilidad de crear un mundo jurídico basado en dos elementos que en otros campos son tan solo aspiraciones: la paz y el bienestar común. 
Aquí se abordará el primer tema sobre el cual debe pronunciarse cualquier rama del Derecho: la definición y la delimitación de su campo de acción, en este caso el espacio ultraterrestre. Se definirá qué se entiende por espacio ultraterrestre, y se expondrán sus límites superior e inferior explicando la importancia de dicha delimitación y su relación con los distintos contextos históricos. Se pretende esgrimir un concepto y una posición frente al tema, basados en los principios que rigen las actividades de los Estados y la exploración del espacio, así como en los postulados de la ciencia, las normas y debates, con el propósito de ser útil al corpus iuris spatialis.

La segunda parte versará sobre la órbita de los satélites geoestacionarios y su caracterización como recurso natural limitado de acceso equitativo. Se plantearán los aspectos y debates principales, y concluirá con una posición que proponga soluciones para los problemas jurídico-políticos que afectan el tratamiento legal de la órbita de los satélites geoestacionarios en nuestro país y en el mundo. Para esto se explicarán conceptos básicos como: órbita, satélite y espectro electromagnético. Asimismo, se recorrerá de forma breve la historia de la teorización y uso de la órbita geoestacionaria, y se explorarán los debates que siguen presentándose con respecto a esta. Se hará un énfasis particular en las discusiones sobre soberanía y el rol de Colombia en la misma, desde la Declaración de Bogotá hasta la Sentencia C-278 de 2004.

\section{DEFINICIÓN Y DELIMITACIÓN DEL ESPACIO ULTRATERRESTRE}

Si hay solamente espacio, sin soles o planetas en él, entonces el espacio pierde su esencia.

\section{Buda}

La odisea del espacio es, en principio, una exploración. Como tal, cuenta con mapas, coordenadas y, por tanto, con una cartografía propia. Toda cartografía es la manifestación del deseo del hombre de conocer sus límites, límites que tienen una importancia fundamental en el campo jurídico: plantear las esferas en las que puede funcionar determinada entidad estatal, y quién es o no es ciudadano de determinado país. El mismo Estado, en sus componentes sustanciales, incluye el territorio dentro del cual puede ejercer su poder exclusivo. Tener claridad sobre cuáles son las fronteras del territorio permite aplicar una norma determinada y los eventuales derechos y deberes que de esta se derivan. Términos como el de propiedad, ciudadanía y soberanía no tienen sentido si no se relacionan de forma directa con una limitación geográfica. Existen así dos categorías generales de límites: los directamente relacionados con la naturaleza (los bordes continentales, los polos, etc.), y los que son puramente abstracciones humanas (las fronteras de las naciones y sus divisiones internas, los territorios marítimos, entre otros).

Es importante establecer desde este momento que cuando hablamos de "espacio" nos referimos al "espacio exterior", o lo mismo, "espacio ultraterrestre". No estaba equivocado en el siglo 
V a. C. el matemático y filósofo griego Tales de Mileto, al afirmar que "lo más grande es el espacio porque lo encierra todo"6, puesto que el término se utiliza para todo aquello comprendido fuera de la atmósfera terrestre. No debe así confundirse el término espacio con el de vacío, porque incluso en los lugares donde no existen cuerpos celestes el espacio posee una muy baja densidad de materia, formada por diversos componentes ${ }^{7}$. Ambas consideraciones, a pesar de no existir una definición oficial de "espacio ultraterrestre", se recuerdan constantemente en la normatividad a través de la expresión “incluso la Luna y otros cuerpos celestes"8.

Inicialmente, delimitar el espacio no tenía sentido, puesto que el hombre aún no había logrado obtener los conocimientos científicotécnicos para explorarlo ${ }^{9}$. Pero los avances científicos y las realidades políticas variaron, y consigo las preocupaciones jurídicas. Sucedió así con los derechos del mar, del aire y de los polos, sus antecesores. En estos, los avances

6 Si bien es cierto que la existencia de Tales de Mileto ha sido cuestión de debate, como ha sucedido con quienes no dejaron trabajos escritos, también lo es que dicho personaje se encuentra varias veces presente en diversos testimonios que lo mencionan. La frase citada se la atribuye a Tales el historiador Diógenes Laercio en el primer tomo de su monumental obra Vidas, opiniones y sentencias de los filósofos más ilustres. Para más referencias sobre este filósofo presocrático se recomienda ver el compendio disponible en línea en: http://www.filosofia.org/cur/pre/talesfyt.htm. (Consultado el 13 de noviembre de 2011).

$7 \quad$ También componen al espacio partículas de elementos como el hidrógeno, neutrinos sin masa, partículas cósmicas, materia oscura, energía oscura y radiación electromagnética, entre otras.

8 Así lo establece toda la normatividad pertinente, incluyendo los Principios jurídicos que rigen la exploración y uso del espacio ultraterrestre incluso la Luna y otros cuerpos celestes, adoptados por la Asamblea General de las Naciones Unidas en 1967.

9 "Generalmente se creyó que para determinar los límites precisos del espacio aéreo y del espacio exterior no era un problema legal que tuviera prioridad en este momento". Informe del Comité ad hoc del Uso Pacífico del Espacio Ultraterrestre, A/4141, del 14 de julio de 1959. en la exploración y la navegación, al igual que los distintos descubrimientos, permitieron que con el tiempo se crearan instituciones como el concepto de aguas internacionales, el derecho aeronáutico y el estatus jurídico del Ártico y el Antártico. De igual forma, los avances científicos permitieron que el hombre iniciara su travesía espacial.

El lanzamiento del Sputnik marcó un hito, pues demostró por primera vez que un ser vivo podía viajar al espacio; desde ese momento y con velocidad meteórica el hombre logró salir de la atmósfera terrestre y pisar otros cuerpos celestes. Las telecomunicaciones se desarrollaron también de forma exponencial, convirtiendo al mundo en lo que es hoy: una sociedad interconectada, en la que la información se comparte y las distancias se derrotan con un solo clic. Este desarrollo se vio especialmente impulsado por la coyuntura sociopolítica de la guerra fría: la competencia armamentista y tecnológica entre la entonces Unión Soviética y los Estados Unidos estimuló una carrera hacia el espacio, en la que la conquista de esa nueva frontera era vista como un triunfo patriótico y prueba de la superioridad del bloque que la obtuviese. Además, las evidentes posibilidades bélicas que planteaba la tecnología espacial eran un gran aliciente para que ambas potencias invirtieran importantes recursos económicos y humanos en su desarrollo. Así, las preocupaciones jurídicas pasaron de la mera posibilidad de violar la soberanía aérea de un país al volar sobre su territorio sin permiso, a viajar por fuera de la atmósfera y llegar a otro punto de la Tierra sin control alguno, manipular las telecomunicaciones y consigo el 
poder, y desarrollar y utilizar armas que exceden grandemente las capacidades defensivas de la mayoría de los países del mundo. Se generó entonces la necesidad de establecer los límites superior e inferior del espacio ultraterrestre y, con ellos, la clarificación del ámbito de aplicación de las normatividades, principios, acuerdos y tratados internacionales sobre el tema.

Si bien la guerra fría terminó con la caída del muro de Berlín, prevalecen los cuestionamientos sobre en qué momento se está violando la soberanía de una nación, y cuándo esta puede reclamar los derechos derivados de esta violación. Estos son asuntos de seguridad nacional que tienen la misma vigencia hoy que hace cincuenta años, y cuya importancia trasciende las posturas que afirman que no es necesaria una delimitación de la frontera espacial porque no ha habido conflictos específicos sobre el tema ${ }^{10}$. No es descabellado decir que uno de los fines del Derecho es prever escenarios y reglamentarlos para evitar inconvenientes; por tanto no debe esperarse a que ocurran conflictos para entrar a regular una delimitación superior e inferior. Así pues, no puede afirmarse de forma ligera que este es un debate puramente teórico y académico ${ }^{11}$.

10 A esta postura se ha aferrado, por ejemplo, los Estados Unidos, a quien ha beneficiado la ausencia de un límite inferior, puesto que muchas veces sus transbordadores navegan a alturas de 50 kilómetros sobre países como Canadá. No debe entonces desconocerse que el limbo jurídico actual plantea beneficios de los que ciertos países pretenden aprovecharse el mayor tiempo posible.

11 En los últimos 20 años los informes de la Comisión sobre Utilización del Espacio Ultraterrestre con Fines Pacíficos han considerado este tópico un problema que, si bien teórico a la fecha, permitiría asegurar el uso del mismo con fines pacíficos. Informe de la Comisión sobre la Utilización del Espacio Ultraterrestre con Fines Pacíficos, $53^{\mathrm{a}}$ periodo de sesiones, 9 al 18 de junio de 2010, Naciones Unidas, Asamblea General; pág. 9, Documento: A/65/20.

\section{El límite superior}

La historia de la ciencia nos ha demostrado que cada vez que el ser humano cree haberlo comprendido todo, aparece un nuevo interrogante, un nuevo hecho o variable que elimina esa certeza. Lo mismo ocurre con el espacio exterior: siempre que el hombre ha intentado establecer su punto máximo ha encontrado otro planeta, otro sistema de planetas u otra galaxia. Si bien existe la posibilidad de que en un futuro y a través de la ciencia el ser humano cuente con herramientas que le permitan verificar un eventual límite del Universo, no es lo que ocurre hoy día, y por lo mismo se afirma que el espacio es infinito, o sea, no tiene límites. Por lo tanto no puede establecerse aún el límite superior del espacio, “(...) a la espera de una explicación más perfecta"12.

\section{El límite inferior: la diferencia entre aire y espacio}

A diferencia del límite superior, el límite inferior del espacio ultraterrestre ha sido cuestión de constante debate. Esto se debe a que, por lejana que sea la distancia entre la Tierra y el objeto que se encuentre en el espacio exterior, no cabe duda de que las normas que aplican sean las pertenecientes al campo del Derecho Espacial.

12 Esta cita es parte de una famosa frase del escritor alemán Thomas Mann, incluida en su ópera prima La Montaña Mágica (1924). Si bien es posible afirmar simplemente que el espacio es infinito, el respeto por la historia de los avances científicos nos obliga a contemplar la posibilidad de un escenario futuro en el que aparezca el límite hasta hoy desconocido. La cita completa dice así: "A pesar de los más desesperados esfuerzos, no se ha podido representar un tiempo finito ni un espacio limitado. Se ha decidido creer que el tiempo y el espacio son eternos e infinitos con la esperanza de conseguir una explicación un poco más perfecta". 
Por el contrario, una ínfima reducción de distancia entre un objeto y la Tierra podría implicar la aplicación de otro campo del Derecho sustancialmente distinto: el Derecho Aeronáutico. De hecho, en ninguno de los dos campos del Derecho existe consenso sobre cuál es el límite, en el primero inferior, y en el segundo, superior o vertical. Al definir el espacio exterior como "todo aquello comprendido fuera de la atmósfera terrestre", estamos distinguiendo el espacio de todo lo terrestre, incluyendo lo que se conoce como "espacio aéreo", o sea aquel espacio de la atmósfera en el que pueden navegar las aeronaves. Esta diferenciación es clave, ya que si el aire y el espacio fuesen exactamente lo mismo, el desarrollo de la aviación habría traído consigo viajes interestelares, el Derecho Espacial y el Derecho Aéreo serían uno, y "el cielo" no sería el límite, puesto que el espacio, como ya se dijo anteriormente, no tiene fronteras.

Existen dos diferencias fundamentales entre ambas ramas del Derecho: la diferenciación entre navegación y desplazamiento, y la aplicación del concepto de soberanía.

\section{La navegación vs. el desplazamiento}

La diferencia fundamental en ambos campos de la ciencia y el Derecho es puramente técnica y se encuentra en que los aviones no son de ninguna forma vehículos espaciales. Los primeros están diseñados para navegar, puesto que el aire es un fluido, y debe "cortarse" para poder movilizarse en él. Por su parte, los vehículos espaciales no navegan sino se desplazan, pues a pesar de que el vacío posee materia, su ínfima densidad obliga a las naves espaciales a tener un medio para adquirir velocidad o propulsarse, y aprovechar la gravedad de los distintos cuerpos celestes para desplazarse ${ }^{13}$. Así, no solo son formas de transportación distintas, sino también fenómenos físicos diferentes que requieren respuestas tecnológicas disímiles. Establecer el límite de acuerdo con el tipo de fenómeno físico se topó, como se verá más adelante, con avances científicos que permitieron crear una especie de fusión entre una aeronave y una nave espacial, como lo es el transbordador.

\section{La aplicación del concepto de soberanía}

La soberanía es el poder exclusivo de un Estado sobre un territorio determinado ${ }^{14}$. En el Derecho Aeronáutico dicho concepto tiene gran importancia puesto que el espacio aéreo es parte del territorio de un Estado, y el ingreso sin permiso de una aeronave en este espacio es una violación a la soberanía y, por tanto, una afrenta contra dicho Estado. Por el contrario, en el Derecho Espacial no existe posibilidad alguna de soberanía ${ }^{15}$, primero, porque sería ilógico que un país reclamara este privilegio en territorios sobre los que no puede ejercerla (ej. las lunas de Júpiter);

13 Vale decir que no todo el espacio comparte esta propiedad. Existen lugares en el espacio que por sus características permitirían la navegación en diversos tipos de fluidos.

14 Es este el concepto más elemental de soberanía. Los propuestos por Hobbes y Rousseau, así como los derivados de estos, tratan de especificidades sobre el ejercicio, la legitimación o el origen del poder y poco aportan a lo que el presente texto busca. Para más sobre el concepto de soberanía en los mencionados autores, se recomienda partir de El Leviatán de Thomas Hobbes o El Contrato Social de JeanJacques Rousseau.

ART. 2 "Tratado sobre los principios que deben regir las actividades de los Estados en la exploración y utilización del espacio ultraterrestre, incluso la Luna y otros cuerpos celestes", 1967. 
segundo, porque la mayoría de los países, así lo quisieran, no cuentan con los medios económicos, científicos y tecnológicos para hacerlo. La diferencia de concepciones y aplicación del concepto de soberanía radica en que, en el caso del Derecho Aeronáutico, la teoría que primó fue la famosa columna de aire del Derecho Romano, mientras que en el Derecho Espacial prevaleció la idea de que el Espacio es, como en el Derecho Marítimo, mare liberum o alta mar ${ }^{16}$. La primera establecía que el hombre era propietario de un terreno determinado, y que este derecho le daba propiedad también sobre todo lo que bajo ese terreno yaciera -minerales, por ejemplo- y todo lo que existiese en la columna de aire comprendida sobre él. Esta teoría no tuvo acogida en el Derecho del Espacio por dos motivos fundamentales: primero, porque la soberanía ad infinitum es lo mismo que soberanía ad absurdum $^{17}$; segundo, porque al girar la Tierra cambiaría constantemente lo comprendido dentro de esa ‘columna de aire' y, por ejemplo, un país podría ser soberano de Saturno en un momento y no serlo al siguiente. Establecer un límite, entonces, puede traer consigo situaciones en gran medida impensables en el Derecho Espacial, y

16 La Convención de las Naciones Unidas sobre el Derecho del Mar, en su artículo 87, establece que: "La alta mar está abierta a todos los Estados, sean ribereños o sin litoral. La libertad de alta mar se ejercerá en las condiciones fijadas por esta Convención, y por las otras normas del derecho internacional. Comprenderá, entre otras, (...) la libertad de navegación, la libertad de sobrevuelo, (...)". Por su parte, el artículo 88 establece que la alta mar será utilizada exclusivamente con fines pacíficos y que ningún estado podrá reclamar soberanía sobre ella. En esto es igual al Derecho del Espacio Ultraterrestre.

17 LACHS, Manfred. El Derecho del Espacio Ultraterrestre. México D.F.: Fondo de Cultura Económica, 1977, pág. 60. Esta posición es compartida por varios autores, entre los que se encuentran G.G. Fenwick, Schöborn y J.L. Brierly. que en gran parte motivan la falta de consenso en el establecimiento de un límite definitivo ${ }^{18}$.

Se acordó entonces que "el espacio ultraterrestre, incluso la Luna y otros cuerpos celestes, no podrán ser objeto de apropiación nacional por reivindicación de soberanía, uso u ocupación, ni de ninguna otra manera" ${ }^{19}$.

\section{Propuestas de delimitación}

Atendiendo a lo anterior, la motivación de delimitar definitivamente el campo de aplicación de uno y otro Derecho ha sido impulsada por miembros de ambas ramas, con el fin de evitar futuros conflictos. Se han presentado varias posiciones y propuestas de delimitación las cuales pueden dividirse, de acuerdo con sus características, en dos grupos: uno con criterio geográfico-científico y otro estrictamente funcional. Todas las posturas tienen pros y contras, los cuales se exponen a continuación:

18 Una de estas situaciones podría ser que determinado país considere que una nave espacial cruzó por su espacio aéreo soberano y por tanto tiene derecho a 'dar de baja' dicha nave, sin considerar que quienes la tripulan son enviados de la humanidad, y que existe un deber de salvamento y rescate, así como un protocolo a seguir respecto a la caída de objetos del espacio, sin importar cuál sea el lugar. También podría presentarse el caso de un objeto que cae del espacio en un país determinado y este decide apoderarse del mismo en vez de regresarlo al país de origen o lanzamiento y reportarlo a las Naciones Unidas. Y, finalmente, otra situación posible sería que un país empezase a cobrar ciertas tarifas a cambio de cruzar su "territorio espacial". Escenarios como estos hay muchos.

19 Artículo II del Tratado sobre los principios que deben regir las actividades de los Estados en la exploración y utilización del espacio ultraterrestre, incluidos la Luna y otros cuerpos celestes (1967), aprobado por la Asamblea General de las Naciones Unidas. Este artículo se conoce como el principio de res communis ómnium del Espacio, el cual es un bien común de toda la humanidad, explotable y aprovechable por todos los países. 
- Criterios geográfico-científicos: esta delimitación se basa en la diferenciación directa entre espacio aéreo y espacio ultraterrestre, por lo tanto, el límite inferior del espacio ultraterrestre sería la atmósfera de la Tierra. Si bien este criterio posee la ventaja sobre los demás de saldar definitivamente el debate, es precisamente en esta ventaja donde radica su debilidad puesto que dicho límite podría no ser flexible frente a los adelantos tecnológicos y científicos ni con la realidad. Estas son algunas de las propuestas para establecer un límite de acuerdo con factores geográficos y científicos.

- Criterio de navegación: hace referencia a la ya explicada distinción entre navegación y desplazamiento, y tiene una relación directa con la aeronáutica. El límite más conocido es la Línea de Kármán, recomendada por la Federación Aeronáutica Internacional ${ }^{20}$. Nombrada en honor a Theodore von Kármán, el ingeniero y físico húngaro-americano que la calculó, la Línea de Kármán se encuentra entre los 83 y los 100 kilómetros de altura. Kármán analizó la relación entre la densidad de la atmósfera y la altitud, y encontró que eran inversamente proporcionales. Así, a mayor altitud menor densidad atmosférica y viceversa. La Línea de Kármán es la altura máxima en la que una nave puede

20 La Federación Aeronáutica Internacional (FAI) es un organismo internacional fundado en 1905 cuya función principal es elaborar la normatividad aplicable a la aeronáutica. Además, la FAI mantiene los registros en el campo de la aeronáutica y regula los deportes aéreos como el vuelo en globo y el aeromodelismo. Para más información visitar el portal www.fai.org “navegar": dícese, puede sostenerse a sí misma viajando en el fluido atmosférico sin sobrepasar la velocidad necesaria para poner un objeto en órbita. En este punto cesa lo que conocemos como aerodinámica y comienza la fuerza centrífuga ${ }^{21}$. La creación y puesta en funcionamiento del transbordador espacial dejó en entredicho esta postura, pues funciona como un vehículo espacial en determinado punto y como una nave en otro, haciendo que la diferenciación no sea tan clara.

- Criterio del límite de la gravedad terrestre: propone establecer como límite la altura en la que cesan de aplicar los efectos e influencia de la gravedad terrestre. Dicho efecto va decreciendo gradualmente y la altura promedio no complace a científicos y técnicos por no ser una frontera claramente discernible.

- Criterio objetivo o de altura específica: esta postura plantea la determinación consensual de una altura específica. Si bien existen múltiples propuestas -una por cada teórico que desee establecer el límite bajo su nombre- y por tanto múltiples alturas ${ }^{22}$, la más conocida es la altu-

21 Dennis Jenkins publicó en la página de la NASA una reflexión interesante sobre el límite del espacio ultraterrestre y qué es un astronauta, en la cual se menciona la Línea de Kármán como límite entre el espacio aéreo y el espacio exterior. Puede consultarse el texto completo en línea, en http://www.nasa.gov/centers/dryden/news/X-Press/ stories/2005/102105_Schneider.html (consultado el 13 de enero de 2012).

22 Entre otros, se ha propuesto el límite atmosférico o de alguna de sus capas (tropósfera, estratósfera, ionósfera, exósfera), o la frontera inferior del espacio o atmósfera meteorológica (80 a $85 \mathrm{Km}$ de altura). Estas propuestas no tienen cabida científica definitiva por cuanto son variables: se entremezclan constantemente y las variaciones 
ra del perigeo o altura mínima de un satélite, la cual es de 100 kilómetros. Esta coincide con la propuesta de la Línea de Kármán en el punto máximo.

- Criterio de capas múltiples: sugiere establecer varias fronteras basadas en fenómenos físicos, al igual que en el derecho marítimo, así: una para delimitar la soberanía territorial, otra donde se hable propiamente del espacio, y una tercera zona neutral y similar a las zonas contiguas ${ }^{23}$.

- Criterios funcionales: esta delimitación se basa en que no debe existir un límite geográfico estricto sino flexible, referente no a una zona en particular sino a la función y objetivos de determinada actividad. Por su flexibilidad, es un criterio con varios seguidores pero no soluciona el debate, simplemente deja abierta la puerta para que el límite sea determinado en cada caso particular.

- Criterio de control eficaz: propone que la soberanía nacional se extienda hasta el punto máximo en el que el Estado pueda resguardarla. Este criterio es poco defendido en razón a que trae implícita una desigualdad entre Estados, basada en la tecnología que estos poseen, especialmente aquella de tipo armamentista. La evidente contradicción entre los principios del

meteorológicas son enormes. LACHS, Manfred, Derecho del Espacio Ultraterrestre, pp. 78 y 79.

LACHS, Manfred. Óp. Cit., p. 79.
Derecho del Espacio Ultraterrestre y esta postura evitan que sea siquiera discutida.

- Criterio propiamente funcional: propone tener en cuenta el "carácter de las actividades de los Estados en el espacio, y en los objetivos que perseguían" ${ }^{24}$. En otras palabras, si la función de determinada nave u objeto es la investigación del espacio y sus componentes, se entiende que aplica el Derecho del Espacio; por el contrario, si su funcionalidad es transportar implementos o personas de un lado al otro del globo, se entiende que es el Derecho Aeronáutico el que aplica.

También existe la propuesta de crear varias fronteras de acuerdo con los distintos tipos de actividades -ej. frontera de investigación, frontera de lanzamiento o puesta en órbita, entre otras-, pero estas fronteras deben atender la tolerancia que tiene cada Estado frente a cada actividad, dícese, queda supeditado a criterios de soberanía.

Siendo que los criterios funcionales no proveen una respuesta definitiva, es en los criterios geográficos y científicos en los que se encuentra la respuesta al debate de la delimitación. Esto va de acuerdo con la necesidad del Derecho de andar mano a mano con la ciencia y de entender las leyes de la naturaleza antes de crear normas sobre las relaciones y actividades humanas. Habiendo resuelto esto, y revisado todo lo anterior, resulta evidente que la forma más lógica y sencilla de establecer el límite inferior

24 Ibidem, p. 80. 
del espacio ultraterrestre es adoptar un límite común, dícese, el criterio objetivo. $Y$ con el fin de erradicar los debates sobre a qué altura específica debería establecerse, es conveniente escoger la altura de 100 kilómetros, sobre la cual existe una especie de consenso tácito entre el Derecho Espacial -en cuanto a la puesta en órbita-, y el Derecho Aeronáutico -sobre el límite máximo de la navegación-. Es pues el límite actual superior de un avión y el perigeo o altura mínima de una nave espacial la línea que debería ser utilizada como delimitación inferior del espacio ultraterrestre. Los cambios tecnológicos que generen nuevas aeronaves y vehículos espaciales que cambien ese punto no serían un problema puesto que, luego de confirmar el límite, este deja de relacionarse a un fenómeno físico como tal, se convierte en una abstracción jurídica referente a una altura determinada y por tanto no tiene por qué variar constantemente. Así, se saldaría de forma definitiva un tema desgastante, dando paso a nuevos debates referentes a las actividades en el espacio y los retos que presenta un límite superior siempre en expansión, como por ejemplo, la posibilidad de vida en otros planetas o el manejo de recursos naturales espaciales.

Por último, debe mencionarse que de acuerdo con el cuerpo jurídico del Derecho del Espacio Ultraterrestre, es la Subcomisión de Asuntos Jurídicos de la Comisión de la Utilización del Espacio Ultraterrestre el ente apropiado para establecer dicho límite en consenso con los países que la conforman. Desafortunadamente, es en el consenso, como en toda decisión democráti- ca, en el que radica el problema que ha evitado la determinación de una frontera cualquiera.

\section{ASPECTOS RELATIVOS A LA ÓRBITA DE LOS SATÉLITES GEOESTACIONARIOS}

\section{Cualquier tecnología suficientemente avanzada es indistinguible de la magia.}

Sir Arthur C. Clarke

Existen aspectos del universo que han despertado nuevas posibilidades tecnológicas. El más importante de todos, a la fecha, ha sido el descubrimiento de la órbita de los satélites geoestacionarios. Esta fue teorizada por el escritor de ciencia ficción y científico Sir Arthur C. Clarke, en su artículo técnico de 1945 titulado "Extraterrestrial Relays". La órbita de los satélites geoestacionarios ha permitido la intercomunicación de todo un planeta en formas previamente inconcebibles, haciendo que toda distancia sea corta y permitiendo compartir casi instantáneamente todo tipo de información. Antes de entrar a discutir sobre los aspectos de la órbita de los satélites geoestacionarios, es pertinente cubrir la definición de tres términos fundamentales para el tema: órbita, satélite y espectro electromagnético.

\section{1. Órbita}

Una órbita puede definirse como la trayectoria de un cuerpo u objeto alrededor de otro, mientras está bajo la influencia de una fuerza central 
(ej., gravedad $)^{25}$. Esta idea proviene de las leyes de los movimientos planetarios planteadas por Kepler, quien además calculó que la Tierra se encuentra en órbita alrededor del Sol, que esta órbita es elíptica y que el Sol es tan solo uno de sus ejes. Es importante establecer desde este instante que las órbitas no existen per se, sino que nacen al momento en que un objeto las recorre. Por tanto, una órbita es, más que todo, una trayectoria, y lo que se conoce como periodo orbital es aquel tiempo en el que un objeto se demora en completar dicha trayectoria (ej. 365 días) ${ }^{26}$.

25

Diccionario llustrado de la Astronomía y Astronáutica. Printer Colombiana Ltda., para Círculo de Lectores S.A. por cortesía de Editorial Everest. Bogotá, 1987, p. 16.

26

Si bien es más común el término "órbita geoestacionaria", no es correcto teniendo en cuenta que es gracias a los satélites geoestacionarios que la órbita existe como trayectoria. Es por esto que el presente texto utiliza la denominación "Órbita de los Satélites Geoestacionarios".
Las órbitas están compuestas de los siguientes elementos:

- Perigeo: es la distancia mínima entre el objeto y su eje. En el caso de la Tierra, la distancia mínima es de 100 kilómetros. (Punto 3 a 2).

- Apogeo: es la distancia máxima a la que puede llegar un objeto bajo la influencia de la fuerza del otro. (Punto 1 a 3). Si se excede esta distancia se comienza un trayecto parabólico.

Eje: es el objeto que ejerce la fuerza. Debe existir uno mínimo, pero pueden ser varios. (Punto 3).

\section{Gráfica 1}

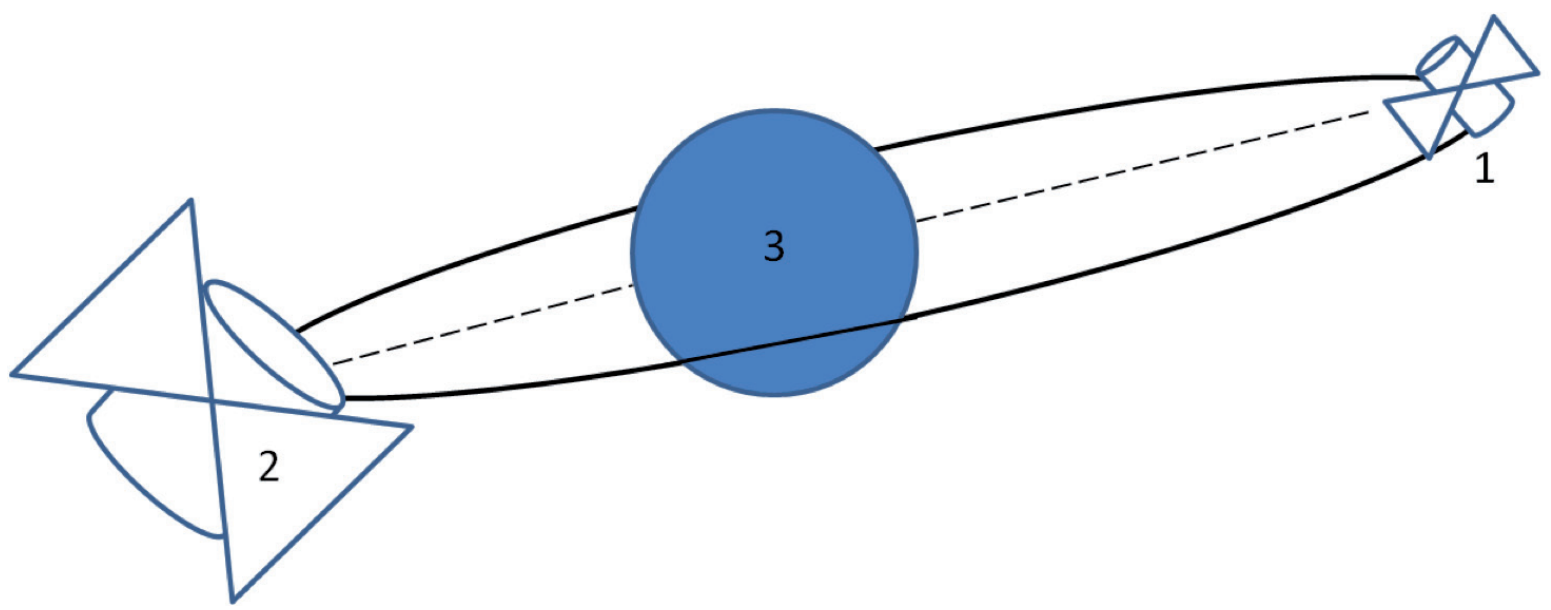


Existen varios tipos de órbitas. Estas pueden categorizarse de acuerdo con sus características. (Ver Gráfica 2).

\section{Gráfica 2}

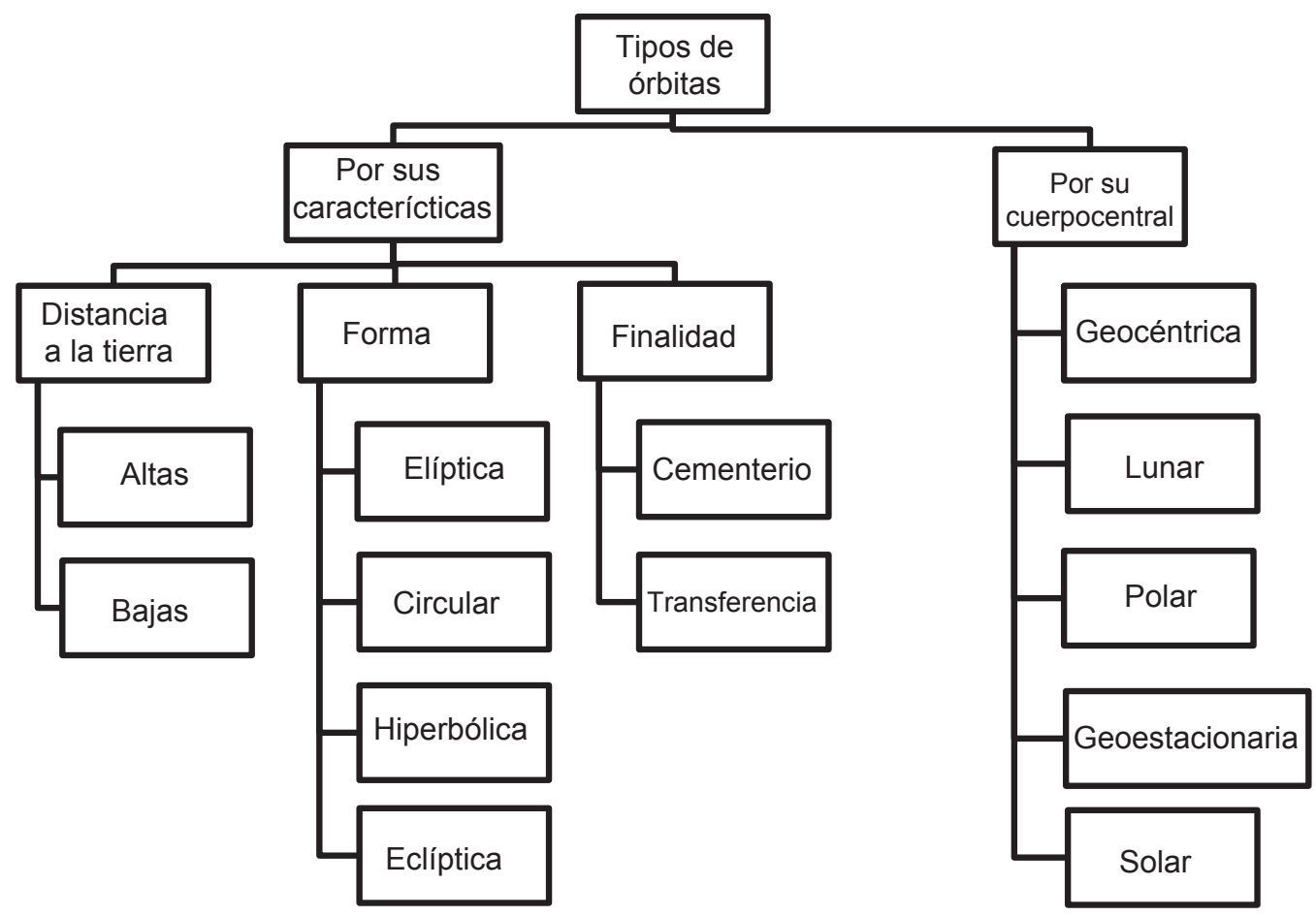

2. Satélites Existen, por supuesto, múltiples clasificaciones adicionales de los satélites, de acuerdo

Son los objetos que orbitan alrededor de otros. Pueden ser naturales (cuerpos celestes que con una gran diversidad de criterios técnicos y científicos $^{27}$.

\section{Espectro electromagnético}

o artificiales (objetos construidos para orbitar alrededor de un planeta u otro objeto). Los satélites siempre se están desplazando a lo largo de las trayectorias denominadas órbitas, y se mantienen 'en órbita' porque llevan la velocidad suficiente para girar. La complejidad que implica poner un satélite artificial en una órbita determinada radica tanto en obtener la altura deseada como en adquirir la velocidad necesaria para que se mantenga en una trayectoria particular.

También conocido como espectro de frecuencias radioeléctricas, el espectro electromagnético puede definirse como la esquematización de la "gama completa de las longitudes de onda de

27 La clasificación de los satélites es similar a la de las órbitas, en cuanto pueden agruparse por sus características físicas (ej. satélite de asteroidales, microsatélites, satélites de energía solar, etc.), su trayectoria (ej. satélites asteroidales, irregulares, etc.), y su finalidad (ej. satélites de comunicaciones, meteorológicos, espías, etc.). 
la radiación electromagnética, desde las ondas de radio a los rayos gamma" 28 . Las ondas representan la energía que emite - 0 'irradia'- un objeto al que se le llama fuente, la cual puede ser desde un electrón hasta una estrella como lo es el Sol. Este esquema cuenta con tres características principales: la longitud de onda, la frecuencia, y la intensidad de la radiación. La longitud de onda es la distancia comprendida entre un punto de una onda y el mismo punto en una onda siguiente. Así, hay ondas cortas

28 Diccionario llustrado de la Astronomía y Astronáutica, p. 119 y largas. La frecuencia es el número de ondas de un punto a otro en un tiempo determinado (usualmente un segundo); se mide en hertzios $(\mathrm{Hz})$, siendo un hertzio una (1) onda por segundo. Entre más alta la frecuencia, mayor número de ondas se emiten por segundo; entre más baja la frecuencia, menos ondas son emitidas en ese mismo lapso de tiempo. Cuando una onda se cruza o comparte trayectoria con otra, y esto ocasiona que sus frecuencias se alteren o eliminen, se produce el fenómeno conocido como 'interferencia'. Por su parte, la intensidad de la radiación es la fuerza con la que se manifiesta la onda.

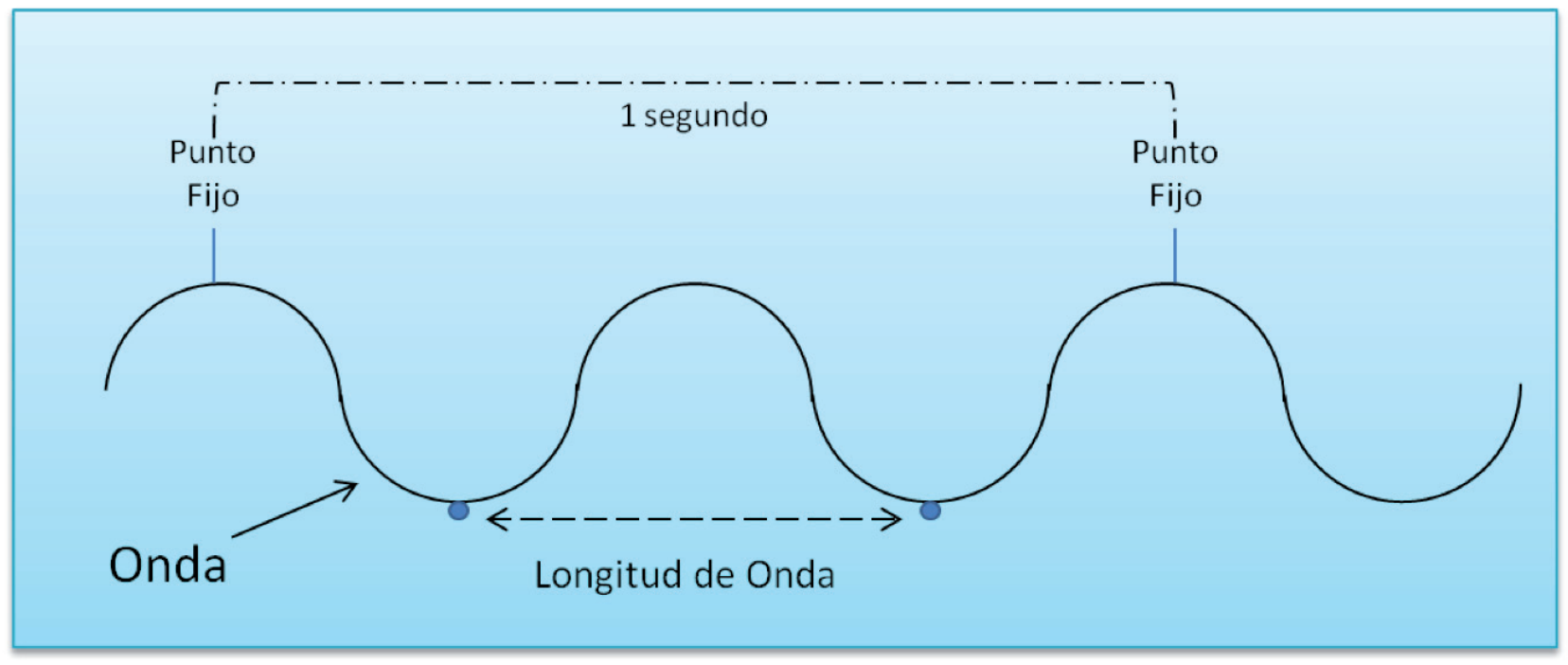

El espectro electromagnético se divide, además, en segmentos o bandas, las cuales a pesar de ser abstracciones inexactas permiten un estudio y un manejo más sencillo de este fenómeno. Entre estas bandas se encuentran las de rayos gamma, la infrarroja, ultravioleta, alta frecuencia, etc.
Habiendo hecho las anteriores aclaraciones, es momento de entrar a discutir lo que se conoce como 'órbita de los satélites geoestacionarios'. Mal denominada 'órbita geoestacionaria' porque, como ya se ha dicho, no existe independientemente de los satélites que la recorren, la órbita de los satélites geoestacionarios es una trayectoria ubicada a 35.786 kilómetros, 
directamente sobre el ecuador terrestre. También se conoce como Órbita o Cinturón de Clarke en honor a su descubridor, y se dice que es 'geosincrónica' puesto que la velocidad de sus objetos va en sincronía con la velocidad de la trayectoria de la Tierra, lo que permite que las fuerzas se neutralicen y los objetos parezcan estacionarios. En otras palabras, el objeto que orbite a dicha altura y siga esa trayectoria, Io hace a la velocidad en la que gira la Tierra. Esta sincronía permite que un satélite de telecomunicaciones ubicado en ella no deba cambiar su direccionamiento para seguir manteniendo una señal de determinada calidad y alcance. Con respecto a la Tierra, este fenómeno es único y natural en el universo, en la forma de un túnel de grosor de aproximadamente 150 kilómetros de ancho, 30 kilómetros de espesor y 360 grados de circunferencia. Gracias a esto, y tal como lo manifestó Clarke en su teorización, se puede emitir una señal con cubrimiento de la totalidad del territorio terráqueo con tres satélites triangulando la Tierra en dicha órbita.

Para que la órbita pueda ser utilizada en forma útil y apropiada, los satélites deben ubicarse a 5 grados de separación o en su defecto utilizar frecuencias distintas; si usan la misma frecuencia deben apuntar a lugares diferentes del planeta. Si no se cumple con estos requisitos se presenta interferencia y, por consiguiente, inutilidad o disminución de las características útiles de los satélites y sus emisiones. Dada la íntima relación existente entre el espectro electromagnético y la órbita geoestacionaria, se denominó al conjunto de ambos Recurso Órbita Espectro (ROE).
Así pues, existen dos limitaciones físicas principales del ROE: la evasión de la interferencia, y la circunferencia y grosor de la órbita. Estas dimensiones y su categoría de única en el universo hacen posible aseverar que es un recurso

\section{natural limitado.}

No es irracional afirmar que el tratamiento jurídico de un recurso natural limitado, así no esté ubicado en nuestro planeta, debe ser distinto al tratamiento del resto de elementos que conforman el espacio ultraterrestre. Este planteamiento motivó varias discusiones, de las cuales la más importante es la relacionada con la soberanía y el acceso equitativo al ROE, de la que Colombia fue precursora.

\section{A. De soberanía a acceso equitativo}

Habiéndose comprobado la utilidad de la órbita de los satélites geoestacionarios y su carácter único, la carrera espacial entre las dos potencias de la guerra fría tomó una nueva dimensión. A pesar de ser aceptado el principio de que el espacio y todo lo que se encuentra en él es un bien común de toda la humanidad, la órbita se convirtió en el recurso ideal para dicha guerra y ambas potencias querían utilizarla para su propio y exclusivo beneficio. La órbita planteaba, entre otras, la posibilidad de espiar al enemigo, de comunicar información de forma instantánea acelerando los operativos militares, o de asentar bases armamentísticas sobre el adversario como amenaza constante. Siendo dueños de una monopólica carrera espacial y bandos opuestos en la guerra fría, los Estados Unidos y la Unión Soviética excluyeron tácitamente del 
debate a los demás países. La lógica, por supuesto, se basaba en que quienes podían utilizar la órbita eran los que debían manifestar un legítimo interés en ella y por lo tanto decidir al respecto.

Impedidos, los demás países se sometieron a esta hegemonía hasta que, en 1974, ante la Asamblea de las Naciones Unidas, sorpresivamente y en contra del principio de res communis ómnium, el embajador colombiano reclamó a nombre de Colombia la soberanía sobre los cinco grados de órbita geoestacionaria ubicados sobre el territorio nacional, correspondientes a la longitud ecuatorial terrestre entre los grados 70 y 75 al oeste de Greenwich. Esto fue sucedido por una reclamación internacional formal, a través del documento conocido como Declaración de Bogotá (1976), en la que varios de los países ecuatoriales ${ }^{29}$, viendo que se abría una puerta para su participación en el debate y aprovechamiento de la órbita, afirmaron considerar los segmentos de la órbita suprayacente a sus fronteras como parte de su territorio nacional. Su reclamación se encontraba justificada, a ojos de estos países, en los siguientes motivos:

a. Que la definición del espacio ultraterrestre no era concluyente y por tanto la órbita no era necesariamente parte de este.

b. Que la normatividad internacional reconocía el ejercicio de la soberanía directa sobre el espacio territorial, entre

29 Los países ecuatoriales que poseen órbita geoestacionaria son: Congo, Gabón, Ecuador, Indonesia, Kenia, Somalia, Uganda, Zaire, Brasil y Colombia. Brasil, efectuando una sabia movida diplomática con miras al futuro político, económico y espacial, firmó la Declaración como observador. otros el Convenio sobre la Aviación Civil Internacional de $1944^{30}$, el Convenio para la Represión del Apoderamiento llícito de Aeronaves -o Convenio de La Haya- de 16 de diciembre de 1970, el Convenio sobre las Infracciones y Ciertos Otros Actos cometidos a bordo de Aeronaves -o Convenio de Tokio - de 14 de septiembre de 1963, y el Convenio para la Represión de Actos Ilícitos contra la Seguridad de la Aviación Civil -o Convenio de Montrealde 23 de septiembre de 1971.

c. Que en concordancia con los dos puntos anteriores, era inaplicable la prohibición contenida en el artículo II del Tratado sobre los Principios que deben Regir las actividades de los Estados en la exploración y utilización del Espacio Ultraterrestre, incluidos la Luna y otros cuerpos celestes. Este punto se ve reforzado en que además, por definición, la órbita geoestacionaria no se comprende o ajusta al anterior Tratado, ni como parte del espacio, ni como cuerpo celeste.

d. Que dicho Tratado no había sido ratificado y por tanto no era de obligatorio cumplimiento.

e. Que el ROE es un recurso natural limitado y como tal debe ser regulado por otros instrumentos internacionales en mira al

30 El Convenio sobre la Aviación Civil Internacional de 1944, más conocido como el Convenio de Chicago, establece en su primer artículo el principio de soberanía absoluta de cada Estado sobre su espacio aéreo. 
desarrollo económico, uso, explotación y acumulación interna de recursos ${ }^{31}$.

f. Que por su posición geográfica estos países corrían un riesgo mayor en cuanto al lanzamiento y caída de objetos, y en consecuencia deberían tener ciertos privilegios sobre los demás.

Así sustentada, la reclamación exigía que cuando se deseara estacionar un satélite en alguno de los grados de la órbita geoestacionaria ubicados sobre el territorio soberano de uno de estos países, debía solicitarse una autorización expresa del país soberano, sin suponer que la autorización per se confería mayores derechos que la mera ubicación.

La comunidad de países rechazó enfáticamente dicha reclamación. Lo hicieron no sólo las potencias que estaban utilizando efectivamente la órbita sino los demás países, especialmente aquellos en vía de desarrollo, relegados ya de la carrera espacial, y que veían aún o más difícil su inclusión si se imponía esta nueva limitación. Ante este rechazo, y conociendo de antemano que esta soberanía nunca iba a ser reconocida por las demás naciones, Colombia cambió el tono de sus reclamaciones aprovechando hábilmente que se había abierto el debate sobre el tratamiento singular que merecía un recurso especial como el ROE. Liderando un planteamiento menos radical -el de la necesidad de crear un sistema jurídico internacional que garantizase el acceso equitativo y el uso eficaz del ROE-,

31 Entre estos instrumentos se pueden citar las Resoluciones 3281 y 2692 aprobadas por la Asamblea General de las Naciones Unidas. se propuso en 1984 que se tuviesen en cuenta las necesidades ya no de los países ecuatoriales sino de los países en desarrollo que, si bien no tenían los recursos para poder utilizar y acceder a la órbita en ese momento, tenían interés e intención de llegar a hacerlo algún día, posibilidad que disminuía rápidamente con la saturación de satélites enviados por las dos potencias predominantes, y uno que otro de la Unión Europea. A esta nueva y más razonable reclamación se unieron gran parte de los países miembros de las Naciones Unidas.

Desde entonces, Colombia sigue liderando los esfuerzos de las naciones que pugnan por la creación y aplicación de un régimen jurídico internacional que defienda los intereses de los países en vía de desarrollo. El sustento jurídico de este alegato se encuentra en los principios de equidad, eficacia y beneficio de toda la humanidad, comprendidos en los distintos tratados que componen el cuerpo jurídico del Derecho del Espacio Ultraterrestre. La diferencia sustancial existente entre los términos igualdad y equidad -el primero de los cuales no es el adoptado en los tratados referentes al Espacio, así como el riesgo inminente de agotar tan valioso recurso-, brindan lógica y legitimidad a estas alegaciones.

\section{B. El dilema del artículo 101 de la Carta Política}

En una postura arcaica, desligada de la realidad jurídica internacional y de la comprensión científica del espacio exterior, y no ajena a debate, nuestra Carta Constitucional incluyó la órbita geoestacionaria dentro de lo que considera el 
territorio nacional junto con los límites fronterizos y las aguas territoriales. El artículo 101 de la Constitución colombiana reza:

“Artículo 101. Los límites de Colombia son los establecidos en los tratados internacionales aprobados por el Congreso, debidamente ratificados por el Presidente de la República, y los definidos por los laudos arbitrales en que sea parte la Nación.

Los límites señalados en la forma prevista por esta Constitución, sólo podrán modificarse en virtud de tratados aprobados por el Congreso, debidamente ratificados por el Presidente de la República.

Forman parte de Colombia, además del territorio continental, el archipiélago de San Andrés, Providencia, Santa Catalina y Malpelo, además de las islas, islotes, cayos, morros y bancos que le pertenecen.

También son parte de Colombia, el subsuelo, el mar territorial, la zona contigua, la plataforma continental, la zona económica exclusiva, el espacio aéreo, el segmento de la órbita geoestacionaria, el espectro electromagnético y el espacio donde actúa, de conformidad con el Derecho Internacional o con las leyes colombianas a falta de normas internacionales." (Subrayado fuera del texto original) $^{32}$

Colombia es una de dos naciones que tuvieron el infortunio de alegar constitucionalmente la soberanía sobre la órbita geoestacionaria. Esto encuentra su explicación no en motivos jurídicos, como los que pretendían hacerse valer en la Declaración de Bogotá, sino en arrebatos nacionalistas y políticos, y en la ignorancia de quienes discutieron y redactaron dicho artículo, equivocación que no pudo ser corregida por los juristas llamados a explicar los aspectos relativos al ROE en el marco del Derecho Espacial. El otro país que incluye este error en su Carta Política es Ecuador.
La aparente incompatibilidad jurídica generada por esta frase fue magistralmente solucionada por la Corte Constitucional en la Sentencia C-278 de $2004^{33}$. Discutiendo la exequibilidad de la Ley 829 de julio 10 de $2003^{34}$, el alto órgano declara dicha ley ajustada a la Carta, basándose en que la última parte del artículo reza: “de conformidad con el derecho internacional o con las leyes colombianas a falta de normas internacionales", deduciéndose así que en el ejercicio de la soberanía sobre la órbita debe prevalecer la normatividad internacional, si bien el texto de la norma: “(...) permite colegir que Colombia ejerce soberanía sobre el segmento de órbita geoestacionaria, en las mismas condiciones en que lo hace respecto (...)" del resto del territorio colombiano. Así, sin transformar el texto constitucional ni traicionar la postura política y diplomática de lucha por un acceso equitativo a la órbita geoestacionaria, el máximo intérprete del texto constitucional integró el nefasto artículo citado al deber de cumplimiento de la normatividad internacional.

33 CORTE CONSTITUCIONAL, Sentencia C-278 de 2004. Referencia: expediente LAT-243. MP: Dr. Marco Gerardo Monroy Cabra y Dr. Manuel José Cepeda Espinosa. Bogotá D. C., 23 de marzo de 2004.

34 "Por medio de la cual se aprueban las Enmiendas al Acuerdo Relativo a la Organización Internacional de Telecomunicaciones por Satélite "INTELSAT", hecho en Washington el 20 de agosto de 1971 y de la Enmienda al Acuerdo Operativo, hecho en Washington el 20 de agosto de 1971, aprobadas por la Vigésima Quinta Asamblea de Partes realizada del 13 al 17 de noviembre de 2000 y la Trigésima Primera Reunión de Signatarios realizada el 9 y 10 de noviembre de 2000, celebradas en Washington, D.C., Estados Unidos de América". 
CONCLUSIÓN: EL ESPACIO EN LA CONSTRUCCIÓN DE UN NUEVO

MUNDO

Este cosmos, que es el mismo para todos, no ha sido hecho por ninguno de los dioses ni de los hombres, sino que siempre fue, es y será un fuego eterno y vivo que se enciende y se apaga obedeciendo a medida.

Heráclito

La faena humana de conquistar el espacio encontró su combustible en el siglo XX. Los avances tecnológicos aparecieron a ritmos insospechados, cambiando la forma de vida de los seres humanos en menos de cincuenta años. Las telecomunicaciones, la investigación, el conocimiento y comprensión del hombre e incluso su propia concepción de su rol en el universo han variado por completo. Sin la exploración espacial el mundo actual sería totalmente distinto. Simultáneamente, el Derecho del Espacio Ultraterrestre también se ha transformado. Como cualquier derecho de desarrollo progresivo, atento a los cambios tecnológicos, científicos y sociales, y a los retos que estos traen consigo, ha debido adaptarse constantemente. A pesar de esto, su ritmo de adaptabilidad no ha sido lo suficientemente rápido. Motivos políticos, más que técnicos o científicos, han generado un estancamiento en aspectos fundamentales como su delimitación. Existen quiénes afirman que este estancamiento también tiene su efecto positivo, al evitar una saturación de normatividades que eventualmente y por su inaplicabilidad ocasionarían la pérdida de valor del régimen jurídico que hoy día constituye el único logro legal basado en la paz y la colaboración entre naciones. Pero si bien esto es cierto, también lo es que el mismo efecto negativo podría obtenerse con la falta de respuesta a problemas, potenciales o reales, y que hay debates que ya deberían estar saldados.

El presente artículo pretendía no sólo narrar problemáticas sino tomar posiciones respecto a las mismas. Se adoptó una postura radical frente a la delimitación del espacio al afirmar que no sólo debe establecerse el límite, sino que además este debe ser a una altura de 100 kilómetros. De igual forma se apoyó la posición de Colombia frente a la órbita de los satélites geoestacionarios, al menos en cuanto a su lucha por buscar espacios para las naciones en vía de desarrollo. Y finalmente, a pesar de ser conscientes del desliz del constituyente, se saldó el debate constitucional sobre la soberanía con la simple y coherente aplicación de la totalidad del sistema normativo y de la norma misma aplicada por la Corte Constitucional.

Sin olvidar que parte de los problemas para llegar a conclusiones como las que aquí se proponen no sólo son políticos sino de desconocimiento sobre el tema, se concluye con la esperanza de haber colaborado en la solución de este inconveniente al resumir brevemente dos de los múltiples aspectos de este nuevo horizonte del Derecho, informando al lector y motivándolo a tomar posturas. Puede que este esfuerzo no aporte en gran medida a la exploración espacial y su desarrollo jurídico, tampoco sobra recordar las palabras del científico y autor 
Carl Sagan al describir con humildad el tamaño del hombre frente al universo entero, afirmando que si bien el infinito es agobiante, también plantea una motivación enorme, puesto que tenemos la conciencia de que el ser humano, por pequeño que sea, es único en la inmensidad. Así, dice Sagan: "El cosmos es todo lo que es, todo lo que fue y todo lo que será. Nuestras más ligeras contemplaciones del cosmos nos hacen estremecer: Sentimos como (sic) un cosquilleo nos llena los nervios, una voz muda, una ligera sensación como de un recuerdo lejano o como si cayéramos desde gran altura. Sabemos que nos aproximamos al más grande de los misterios".

\section{Bibliografía}

El universo es hostil sólo cuando usted no conoce sus leyes.

Para aquellos que las conocen y obedecen, el universo es amigable.

Wernher von Braun

CORTE CONSTITUCIONAL, Sentencia C-278 de 2004. Referencia: expediente LAT-243. MP: Dr. Marco Gerardo Monroy Cabra y Dr. Manuel José Cepeda Espinosa. Bogotá D. C., 23 de marzo de 2004.

Círculo de Lectores. Diccionario Ilustrado de la Astronomía y Astronáutica. Bogotá: Printer Colombiana Ltda., 1987, pág. 16.
Federación Aeronáutica Internacional. www.fai. org

http://www.filosofia.org/cur/pre/talesfyt.htm. (Consultado el 13 de noviembre de 2011).

JAMESON, Fredic. Arqueologías del futuro: El deseo llamado utopía y otras aproximaciones de ciencia ficción. Madrid: Akal, 2009.

JENKINS, Dennis. A Word about the definition of space. http://www.nasa.gov/ centers/dryden/news/X-Press/stories/2005/102105_Schneider.html (consultado el 13 de enero de 2012).

LACHS, Manfred. El Derecho del Espacio Ultraterrestre. México D.F.: Fondo de Cultura Económica, 1977.

Naciones Unidas, Asamblea General. "Cuestionario sobre posibles cuestiones jurídicas relacionadas con los objetos aeroespaciales: respuesta de los Estados miembros". Comisión sobre la Utilización del Espacio Ultraterrestre con Fines pacíficos, DOCUMENTO: A/AC.105/635 y AD. 1- 10, 15 de febrero de 1996.

Naciones Unidas, Asamblea General. “Informe de la Comisión sobre la Utilización del Espacio Ultraterrestre con Fines Pacíficos". 53a periodo de sesiones, 9 al 18 de junio de 2010.

Naciones Unidas, Asamblea General. “Informe de la Subcomisión de Asuntos Jurídicos sobre su $50^{\mathrm{a}}$ periodo de sesiones, celebrado en Viena del 28 de marzo al 8 de abril 
de 2011". Comisión sobre la Utilización del Espacio Ultraterrestre con Fines Pacíficos, Subcomisión de Asuntos Jurídicos, DOCUMENTO: A/AC.105/990, 20 de abril de 2011.

Naciones Unidas, Asamblea General. “Leyes y prácticas nacionales relacionadas con la definición y la delimitación del espacio ultraterrestre". Comisión sobre la Utilización del Espacio Ultraterrestre con Fines Pacíficos, 27 de enero de 2006.

Naciones Unidas, Asamblea General. “Leyes y prácticas nacionales relacionadas con la definición y la delimitación del espacio ultraterrestre". Comisión sobre la Utilización del Espacio Ultraterrestre con Fines Pacíficos, 2 de febrero de 2009.

Naciones Unidas, Asamblea General. “Leyes y prácticas nacionales relacionadas con la definición y la delimitación del espacio ultraterrestre". Comisión sobre la Utilización del Espacio Ultraterrestre con Fines Pacíficos, 19 de enero de 2011.

Naciones Unidas, Asamblea General. “Leyes y prácticas nacionales relacionadas con la definición y la delimitación del espacio ultraterrestre". Comisión sobre la Utilización del Espacio Ultraterrestre con Fines Pacíficos, 9 de diciembre, 4 de marzo y 11 de enero de 2010.

Naciones Unidas, Asamblea General. “Leyes y prácticas nacionales relacionadas con la definición y la delimitación del espacio ultraterrestre". Comisión sobre la Utilización del Espacio Ultraterrestre con Fines Pacíficos, 21 de enero 2008.

Naciones Unidas, Asamblea General. “Leyes y prácticas nacionales relacionadas con la definición y la delimitación del espacio ultraterrestre". Comisión sobre la Utilización del Espacio Ultraterrestre con Fines Pacíficos, 8 de febrero de 2007.

Naciones Unidas, Asamblea General. “Preguntas relativas a la definición y delimitación del espacio ultraterrestre: respuestas recibidas de los Estados Miembros", Comisión sobre la Utilización del Espacio Ultraterrestre con Fines Pacíficos, 21 de enero de 2008; “Leyes y prácticas nacionales relacionadas con la definición y la delimitación del espacio ultraterrestre", Comisión sobre la Utilización del Espacio Ultraterrestre con Fines Pacíficos, 20 de marzo de 2006.

Naciones Unidas, Asamblea General. “Proyecto de informe del Presidente del Grupo de trabajo sobre la definición y delimitación del espacio ultraterrestre". Comisión sobre la Utilización del Espacio Ultraterrestre con Fines Pacíficos, Subcomisión de Asuntos Jurídicos, $50^{a}$ periodo de sesiones, Viena, 28 de marzo a 8 de abril de 2011.

Naciones Unidas, Asamblea General. “Proyecto de informe: Cuestiones relativas a la definición y delimitación del espacio ultraterrestre y el carácter y utilización de la órbita geoestacionaria, incluida la consideración de medios y arbitrios para asegurar la utilización racional 
y equitativa de la órbita geoestacionaria, sin desconocer el papel de la Unión Internacional de Teleco municiones". Comisión sobre la Utilización del Espacio Ultraterrestre con Fines Pacíficos, Subcomisión de Asuntos Jurídicos, $50^{\text {a }}$ periodo de sesiones, Viena, 4 de abril de 2011.

Naciones Unidas, Asamblea General. "Tratado sobre los principios que deben regir las actividades de los Estados en la exploración y utilización del espacio ultraterrestre, incluso la Luna y otros cuerpos celestes", 1967.

Naciones Unidas, Asamblea General. Informe de la Comisión sobre la Utilización del Espacio Ultraterrestre con Fines Pacíficos". Documento: A/65/20, 53 ${ }^{\mathrm{a}}$ periodo de sesiones, 9 al 18 de junio de 2010.
Naciones Unidas, Asamblea General. Informe del Comité ad hoc del Uso Pacífico del Espacio Ultraterrestre. Documento A/4141, del 14 de julio de 1959.

SÁNCHEZ, Guillermo, GALLEGO, Eduardo. "Qué es la ciencia ficción?" http://www.ciencia-ficcion.com/opinion/op00842.htm\# (Consulta: 11 de diciembre de 2011).

Unión Internacional de Telecomunicaciones. "Conjunto de textos fundamentales de la Unión Internacional de Telecomunicaciones adoptados por la Conferencia de Plenipotenciarios". 2011. 